REVIEW

\title{
Clinical Significance of High-intensity Zone for Discogenic Low Back Pain : A Review
}

\author{
Subash C. Jha, Kosaku Higashino, Toshinori Sakai, Yoichiro Takata, Mitsunobu Abe, Kazuta Yamashita, \\ Masatoshi Morimoto, Shoji Fukuta, Akihiro Nagamachi, and Koichi Sairyo \\ Department of Orthopedics, Tokushima University, Tokushima, Japan
}

\begin{abstract}
High-intensity zone (HIZ) was originally described as a high-intensity signal on T2-weighted magnetic resonance (MR) images, located in the posterior annulus fibrosus, clearly separated from the nucleus pulposus. Among symptomatic patients with low back pain, $\mathrm{HIZ}$ is present in $\mathbf{2 8 - 5 9 \%}$ of cases. In morphologically abnormal discs, high sensitivity and specificity of $81 \%$ and $79 \%$, respectively, were reported for HIZs and concordant pain during discography. In contrast, another report indicated low rates. Although most papers reported high sensitivity and specificity for this relationship, it remains controversial. Regarding the pathology of HIZs, inflammatory granulation tissues are found at sites showing HIZs. Such inflammatory tissues produce pro-inflammatory cytokines and mediators, which sensitize the nociceptors within the disc and cause pain. An effective treatment for this condition is yet to be established. Recently, minimally invasive surgery using percutaneous endoscopic discectomy (PED) under local anesthesia was introduced. After removal of the degenerated disc material, the HIZ is identified with the endoscope and then coagulated and modulated with a bipolar radio pulse. This technique is called thermal annuloplasty. In conclusion, HIZs is an important sign of painful intervertebral disc disruption, if identified precisely based on factors such as location and intensity. J. Med. Invest. 63 : 1 - 7, February, 2016
\end{abstract}

Keywords : High-intensity zone, Magnetic resonance images, Discography, Percutaneous endoscopic discectomy, Thermal annuloplasty

\section{INTRODUCTION}

High-intensity zone (HIZ) has been described as an important sign visualized on magnetic resonance (MR) images for the diagnosis of internal disc disruption causing discogenic low back pain (DLBP). However, its identification in asymptomatic individuals has made it a controversial issue. This report discusses recent and changing trends in the diagnostic significance and management of HIZs.

\section{BACKGROUND}

In approximately $85 \%$ cases with low back pain, the specific cause remains unidentified $(1,2)$. More than $40 \%$ of cases with chronic LBP are attributed to intervertebral disc damage, of which over $70 \%$ of cases have no nerve root compression (3-6). DLBP due to internal disc disruption is considered to be the most common cause for chronic LBP $(4,5)$. Since clinical examination alone is not capable of identifying the exact source of chronic LBP, various investigations and related signs have been identified in the last few decades $(7,8)$. Provocative discography, first described by Lindolm and Hirsch in 1948, provides information on the morphological characteristics of the disc as well as the provoked pain response $(9,10)$. Hence, it is still considered the gold standard by many clinicians for the diagnosis and management of DLBP. However, provocative discography remains controversial due to its invasive nature and associated complications, such as infection,

Received for publication July 20, 2015 ; accepted August 17, 2015.

Address correspondence and reprint requests to Koichi Sairyo, MD, $\mathrm{PhD}$, Department of Orthopedics, Tokushima University 3-18-15 Kuramoto, Tokushima 770-8503, Japan and Fax : +81-88-633-0178. neurological injury, and possible contrast medium reaction. Moreover, the interpretation of the results is strongly influenced by the psychological condition of the patient $(11,12)$. For this reason, many clinicians have reservations regarding the significance and utility of provocative discography in the diagnosis of DLBP.

A significant breakthrough was made in 1992, when, for the first time, Aprill and Bogduk described HIZs on MR images for diagnosing DLBP (13). Their study demonstrated a prevalence of $28.6 \%$ for HIZs, with sensitivity, specificity, and positive predictive value (PPV) of $71 \%, 89 \%$, and $86 \%$, respectively, for diagnosing symptomatic LBP patients (13). After that, various studies have investigated the significance of HIZs : some reports have described a limited role of HIZs in the diagnosis of LBP due to low sensitivity and high prevalence in asymptomatic subjects, while many other reports have considered HIZs to be a reliable non-invasive means of confirming painful internal disc disruption.

Figure 1 shows typical MR images in a patient with DLBP. This patient is a 36 -year-old male baseball player who experienced LBP on flexion while playing. On sagittal and axial MR images (Figure 1a, 1b), the HIZ is obvious at the L4-5 level, with concordant pain on provocative discography (Figure 1c). When he experienced severe pain, he was treated by intradiscal injection. He was able to play through the season by repeating the intradiscal injection therapy. At the end of the season, he retired from professional baseball.

\section{DEFINITION AND EPIDEMIOLOGY}

Originally, HIZ was defined as a high-intensity signal on T2weighted MR images, located posteriorly in the annulus fibrosus, which is clearly dissociated from the signal of the nucleus pulposus (13). However, most authors now believe that a similar lesion occurring at the posterolateral annulus fibrosus should also be 

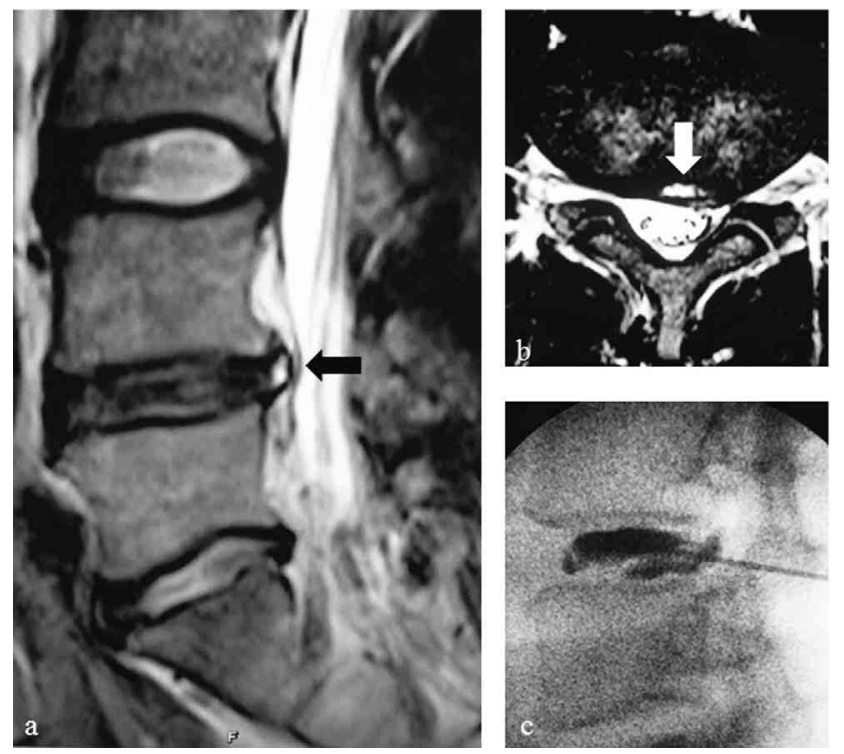

Figure 1. (a) Sagittal and (b) axial T2-weighted magnetic resonance (MR) images showing high-intensity zones (HIZs) within the posterior annulus fibrosus at the L4-5 level (arrows). (c) Positive on discography.

considered as HIZ, and such lesions are usually included in related studies (14-18). Bogduk (19) and Liu et al. (18) emphasized that HIZ must be an intense signal, rather than any spot, in the posterior annulus.

The prevalence of HIZs ranges from $28 \%$ to $59 \%$ in patients with LBP, but its prevalence has also been reported to be as high as $56 \%$ in patients without LBP (Table 1). In a prospective study of 144 patients, Lam et al. (20) concluded that HIZs are a reliable indicator of symptomatic annular tears causing pain, with prevalence up to $51 \%$. Their findings were comparable to the results of Aprill and Bogduk (13). Wang et al. (16) reported that among 623 patients, $32.1 \%$ exhibited an HIZ in at least one disc, and the LBP rate among the patients with HIZs was significantly higher than that among the patients without HIZs $(57.5 \%$ vs. $47.8 \%, \mathrm{P}<0.05)$. In a prospective comparative study conducted by Liu et al. (18), the prevalence of HIZs was $45.8 \%$ in symptomatic LBP patients but $20.2 \%$ in the asymptomatic control group.

Table 1 : Prevalence of HIZs in symptomatic and asymptomatic populations.

\begin{tabular}{|l|l|l|l|}
\hline Study & $\begin{array}{l}\text { Symptomatic } \\
(\%)\end{array}$ & $\begin{array}{l}\text { Asymptomatic } \\
(\%)\end{array}$ & No. of patients \\
\hline Aprill and Bogduk (13) & 28 & N/A & 500 \\
\hline Liu et al. $(18)$ & 45.8 & 20.2 & $72 / 79$ \\
\hline Lam et al. $(20)$ & 51 & N/A & 144 \\
\hline Rankine et al. $(21)$ & 45.5 & N/A & 156 \\
\hline Mitra et al. $(22)$ & 28.6 & N/A & 650 \\
\hline Carragee et al. $(23)$ & 59 & 24 & 42 \\
\hline Weishaupt et al. $(24)$ & N/A & 32 & 60 \\
\hline Stadnik et al. $(25)$ & N/A & 56 & 36 \\
\hline
\end{tabular}

Rankine et al. (21) assessed 156 patients with LBP and concluded that HIZs occurred at a prevalence of $45.5 \%$, but its occurrence did not define a group of patients with specific clinical features.
Mitra et al. (22) found a $28.6 \%$ prevalence of HIZs, but concluded that the correlation between HIZs and symptomatic changes was not statistically significant. Carragee et al. (23) noted that the prevalence of HIZs was $59 \%$ in a symptomatic group of patients and $24 \%$ in the asymptomatic group. Therefore, they questioned the meaningful clinical use of this sign in the diagnosis of DLBP. Weishaupt et al. (24) and Stadnik et al. (25) found the prevalence of HIZs to be $32 \%$ and $56 \%$ in asymptomatic subjects, respectively. Factors such as study population, size of the study cohort, technical factors related to MR imaging, and the subjective nature of the assessment of HIZs may have caused the difference in the prevalence of HIZs reported in these studies (24).

\section{CORRELATION WITH DISCOGRAPHY}

Studies have demonstrated that in morphologically abnormal discs, a significant correlation exists between HIZ-positive discs and exact or similar pain reproduction on provocative discography (Table 2). In a retrospective study, Saifuddin et al. (14) reviewed 58 patients based on MR images and discography findings. Of 152 discs injected on discography, they identified 86 annular tears, of which 70 were associated with concordant pain provocation. On MR images, HIZs were identified in 27 discs, of which 24 were associated with pain reproduction on provocative discography. The specificity, PPV, and negative predictive value (NPV) of MR images in diagnosing a concordantly painful annular tear were $95.2 \%, 88.9 \%$, and $47 \%$, respectively. The sensitivity was only $26.7 \%$, which was lower than the findings of Aprill and Bogduk. However, Lam et al. (20) conducted a study of 144 patients, and reported findings similar to those of Aprill and Bogduk, with sensitivity, specificity, and PPV of $81 \%, 79 \%$, and $87 \%$, respectively, for HIZs and concordant pain in morphologically abnormal discs. Schellehas et al. (15) assessed 63 patients and found that 87 of 100 discs with HIZs were concordantly painful on discography, with PPV and NPV of $87 \%$ and $97 \%$, respectively. In a retrospective study by Smith et al. (27) the sensitivity and specificity of HIZs and concordant painful discs were $31 \%$ and $90 \%$ respectively, but PPV was relatively low at $40 \%$, suggesting that HIZs may not be indicative of internal disc disruption. Although some reports have observed a low rate of correlation between HIZ and concordant pain in discography, the majority of reports have shown good agreement with the report of Aprill and Bogduk (13) indicating that these two parameters are positively correlated. This issue remains controversial.

Table 2 : Correlation between HIZs and exact or similar pain reproduction on discography.

\begin{tabular}{|l|l|l|l|l|}
\hline Study & $\begin{array}{l}\text { Sensitivity } \\
(\%)\end{array}$ & $\begin{array}{l}\text { Specificity } \\
(\%)\end{array}$ & $\begin{array}{l}\text { PPV } \\
(\%)\end{array}$ & $\begin{array}{l}\text { NPV } \\
(\%)\end{array}$ \\
\hline Ito et al. $(5)$ & 52.2 & 95.6 & 60 & N/A \\
\hline Aprill and Bogduk (13) & 71 & 89 & 86 & N/A \\
\hline Saifuddin et al. $(14)$ & 26.7 & 95.2 & 88.9 & 47 \\
\hline Schellhas et al. $(15)$ & N/A & N/A & 87 & 97 \\
\hline Lam et al. $(20)$ & 81 & 79 & 87 & N/A \\
\hline Hebelka et al. $(26)$ & 49 & 69 & 70 & 76 \\
\hline Smith et al. $(27)$ & 31 & 90 & 40 & N/A \\
\hline
\end{tabular}

\section{PATHOGENESIS}

In the literature, discogenic pain is described as LBP with or without leg pain, caused by disc degeneration and/or annular rupture 
(28-30). Potential causes of non-discogenic LBP include spondylolisthesis, spinal stenosis, degenerative scoliosis, disc herniation, spinal fracture, infection, and neoplasm. Routine MR imaging of the lumbosacral spine for investigating the cause of DLBP can reveal various abnormalities of the disc, but the presence of an abnormality does not necessarily indicate that it is responsible for the pain $(24,31)$.

In normal adults, the annulus fibrosus is innervated by the recurrent meningeal nerve and by branches from the ventral ramus of the somatic spinal nerve (32). Most of the nerve supply of the intervertebral disc is limited to the periphery of the annulus fibrosus (32-34). With this knowledge, Sach et al. (35) found that annular tears extending to the inner third of the annulus fibrosus were asymptomatic, whereas tears that extended to the peripheral third produced pain in $70 \%$ of their patients. Since the posterior annulus is considered structurally weak and experiences high stress concentrations, this site is more vulnerable to disruption and HIZs compared with the anterior annulus (36).

Various authors have proposed that HIZs comprise fluid-filled zones, possibly due to a detached nucleus pulposus that is trapped between the lamellae of a torn annulus fibrosus, following secondary inflammation that results in edema, causing the characteristic signal abnormality on MR images $(13,15,37,38)$. This area appears to enhance on gadolinium DTPA-MR images, indicating the presence of granulation tissue or neovascularization induced by inflammation (39). This was supported by the results of a cadaveric study by Yu et al. (40), which demonstrated that radial and transverse tears could be identified on MR images, as well as a histological study by Peng et al. (41) on lumbar intervertebral discs containing HIZs in the posterior annulus. Such inflammatory granulation tissue produces pro-inflammatory cytokines and mediators, which sensitize the nociceptors within the disc, causing pain $(8$, 41). These findings suggest that biomechanical mediators are more important than mechanical compression alone in the pathogenesis of back pain $(8,17)$.

\section{INFLUENCE OF THE MEASUREMENT CONDITIONS AND STRENGTH OF MAGNETIC FIELD}

Originally, HIZ was identified and defined on T2-weighted MR pulse sequence (13), and most of the studies about HIZ have used 1.5 Tesla $(\mathrm{T})$ superconducting magnet in common $(14-16,18,20$, $22,27)$. It is not clear whether strength or any other sequence of MR imaging influences the detection of HIZ. It is considered that more powerful MR imaging system has more resolution, making the detection of HIZ easier and effective (20). Pande et al. (42) used $0.5 \mathrm{~T}$ system for evaluating significance of HIZ in 200 patients. They demonstrated prevalence of HIZ was $13 \%$ and $17 \%$ according to observer A and B respectively, without any clinical significance for diagnosis of disrupted and painful disc. Similarly, only $27 \mathrm{HIZs}$ were identified in MR images, out of 152 discs (17.7\%) examined by Saifuddin et al. (14) using 0.5-1.5 T system. The exact numbers of patient examined by $0.5 \mathrm{~T}$ or $1 \mathrm{~T}$ or $1.5 \mathrm{~T}$, was not included.
Rankine et al. (21) demonstrated prevalence of HIZ as high as $45.5 \%$ using $1 \mathrm{~T}$ system in their study. Hence, it is difficult to comment the significance of strength of MR imaging for detection of HIZ. We believe $1.5 \mathrm{~T}$ system frequently used worldwide is optimum strength for detection of HIZ.

Sagittal and axial T2-weighted sequences with slice thickness from 3-5 $\mathrm{mm}$ and interspace gap ranging from 0.4-1.5 $\mathrm{mm}$ have been used in majority of studies of HIZs (14-16, 18, 20, 22, 27, 51). Use of short tau inversion recovery (STIR) MR sequence can be helpful in HIZ identification but it will be too early to comment so, without any relevant clinical study. Liu et al. (18) in their study conducted MR study at about 3:00 PM to minimize the effect of water influx and hydration of the discs, causing increase in disc signal as much as $25 \%$ on $\mathrm{T} 2$-weighted images.

\section{LOCATION}

More than two-third of HIZs are identified at lower lumbar segments, with the highest number being identified at L4-5, followed by L5-S1 (Table 3). Wang et al. revealed that the prevalence of multisegmental HIZs (Figure 2) was 16.5\%, and 25 of 33 cases with multisegmental HIZs exhibited HIZs in an adjacent disc (16). In the same study, HIZs were more frequently observed in the inferior part of the annulus fibrosus (superior-middle-inferior ratio, $39: 59: 140$ ), with statistically significant differences (16). Thus, along with the original definition for the location of HIZs, which included lesions in only the posterior annulus fibrosus, many authors now think that HIZs exist circumferentially and that the original definition should be expanded to include non-midline lesions, while keeping in mind that posterior lesions are the commonest (15-17).

\section{INTENSITY}

With growing controversies surrounding the definition of HIZ and its significance, an important factor is the identification of true HIZs. Failure to distinguish true HIZs from low/medium intensity zones may lead to a lower diagnostic value of HIZs (44). Reliable and sophisticated measures are needed for the precise detection and assessment of HIZs to improve the clinical utility of this finding (26). O'Neil et al. (45) divided HIZs into grades of mild, moderate, and marked hyperintense. They found that moderate and marked lesions had higher specificities. Carragee et al. (23) found that if the signal was within $10 \%$ of the cerebral spinal fluid (CSF) intensity, it could be considered as a true HIZ, but they did not discuss reliability. In a quantitative study conducted by Liu et al. (18), the mean signal of HIZs was significantly brighter in symptomatic subjects than in asymptomatic subjects. Therefore, they defined a true $\mathrm{HIZ}$ as a lesion with signal intensity at least as bright as $50 \%$ of the CSF signal intensity. In their study, the corresponding disc with HIZs was outlined first on T1-weighted MR images. The contour of the disc obtained on T1-weighted MR images was copied

Table 3 : Distribution of HIZs in different lumbar intervertebral discs.

\begin{tabular}{|l|l|l|l|l|l|l|}
\hline Study & L1-2 & L2-3 & L3-4 & L4-5 & L5-S1 & Total no. of HIZs \\
\hline Saifuddin et al. $(14)$ & 0 & 2 & 3 & 14 & 8 & 27 \\
\hline Schellhas et al. (15) & 2 & 1 & 14 & 46 & 37 & 100 \\
\hline Wang et al. (16) & 7 & 10 & 40 & 106 & 75 & 238 \\
\hline Lam et al. $(20)$ & 0 & 2 & 13 & 44 & 32 & 91 \\
\hline Ricketson et al. $(37)$ & 0 & 0 & 0 & 7 & 2 & 9 \\
\hline Wilkens et al. (43) & 0 & 0 & 5 & 9 & 9 & 23 \\
\hline
\end{tabular}




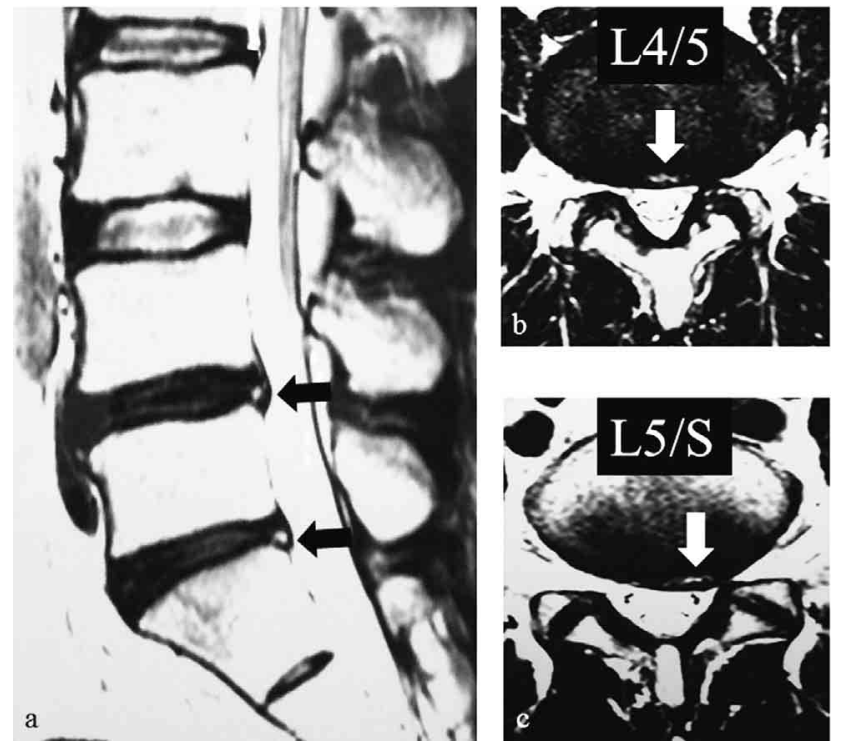

Figure 2. (a) Sagittal and (b and c) axial T2-weighted MR images showing multisegmental HIZs within the posterior annulus fibrosus of LA5 and L5-S1 levels (arrows).

and moved to T2-weighted MR images, with some fine adjustments. A clear sample of CSF located adjacent to the disc was used as the reference to adjust signal intensity. The CSF-adjusted HIZ was a ratio of the mean signal intensity of the HIZ to that of the adjacent CSF. If the adjacent spinal canal was too narrow to obtain an adequate sample of CSF, the nearest level possible was taken as the reference point for adjusting the signal intensity. Liu et al. also recommended this method as a more practical protocol to increase measurement precision and reduce error in both clinical and radiological studies, with both excellent intra- and inter-observer reliability (18). All these findings support the Bogduk hypothesis : A "low/medium intensity zone" representing an annular fissure can be found in MR images in asymptomatic populations, whereas the symptomatic population will have true HIZs, which are significantly brighter and are a reliable marker of discogenic pain $(18,19)$.

\section{TREATMENT}

Various treatment modalities have been described based on the consideration of HIZs as a potential source of DLBP. In a longitudinal study, Mitra et al. (22) concluded that HIZs do not change, spontaneously resolve, or improve in most patients over time and that there is no statistically significant relationship between the evolution of HIZs and changes in symptoms. However, various medical and minimally invasive surgical techniques for treating HIZs and DLBP are presently under trial, with varying clinical results.

In a randomized controlled trial by Wilkens et al. (43), oral glucosamine intake for 6 months did not alter the presence of HIZs as compared with placebo. In their study, HIZs resolved in 3 of 23 discs in 3 of 21 patients : 1 of 8 patients on oral glucosamine therapy and 2 of 13 patients on placebo. The idea behind using glucosamine therapy is to target interleukin (IL) $-1 \beta$ (46), considering that HIZ is a potential marker of an osteoarthritic degenerative process and is related to the secretion of pro-inflammatory mediators (8), and that HIZs may contain IL-1ß. Previous research has also demonstrated the inability of glucosamine to reduce LBP or
LBP-related disability (47).

Various minimally invasive procedures, such as intradiscal electrothermal therapy (IDET), intradiscal injections, and percutaneous endoscopic discectomy and thermal annuloplasty (PEDTA) have been assessed. Promising effects have been observed but no clear conclusions have been reached (48-50). While these therapies offer satisfactory symptomatic relief from LBP, as noted by treating clinicians, their mechanisms of action have yet to be comprehensively explained. Narvani et al. (51) reported symptomatic improvement in 8 of 10 patients treated with IDET at 6 months postprocedure, though the HIZs were noted to persist even after the procedure. They postulated three possible explanations for their findings : (1) the presence of HIZ does not accurately predict annular tear ; (2) IDET does not seal the annular tear but rather stiffens the annulus and offloads the pain-sensitive areas; and (3) IDET seals the tears but does not reverse the chronic inflammatory changes of HIZs.

In a prospective study, Miller et al. (48) suggested that intradiscal injection of a solution consisting of $50 \%$ dextrose and $0.25 \%$ bupivacaine may have a place in the management of pain arising from advanced lumbar degenerative disc disease. Since oral glucosamine was ineffective, Derby et al. (49) compared IDET with intradiscal restorative injections consisting of injectable glucosamine and chondroitin sulfate combined with hypertonic dextrose and dimethylsulfoxide to reduce pain and disability in patients with chronic DLBP. The results were similar for intradiscal injections and IDET, but the injections provided a better cost-benefit ratio. IDET was performed in 74 patients, while 35 patients received intradiscal injection; while satisfactory results were noted in both procedures, outcomes were slightly better for the injections than those for IDET. Moreover, only $47.8 \%$ of the patients who underwent IDET reported improvement, whereas $65.6 \%$ of the patients who received intradiscal injection were satisfied with the outcome.

Percutaneous endoscopic discectomy (PED) was initially established as a novel surgical technique for the management of lumbar disc herniation (52). It is a minimally invasive procedure, where an endoscope is inserted intradiscally via the posterolateral, transforaminal approach through an $8 \mathrm{~mm}$ skin incision under local anesthesia, causing minimum injury to the back muscles $(52,53)$. Additionally, radiofrequency thermal annuloplasty (TA) with PED (PEDTA) has been reported as the most recent advancement in the treatment of DLBP (54-56).

In a retrospective study of 113 patients of DLBP treated by PEDTA, Tsou et al. (54) reported 73.5\% satisfactory outcomes (excellent $15 \%$, good $28.3 \%$, and fair $30.1 \%$ ) at 2 -year follow-up, while $26.5 \%$ patients were found to have poor results. Sairyo et al. (55) reported the cases of 4 professional athletes with DLBP, including 2 showing HIZs on MR images, who were successfully treated by PEDTA. The endoscopic visualization of vascularized granulation tissue in the outer region of the annulus fibrosus, which correlated with the location of HIZs on MR images, further supports the significance of HIZs and helps in radiofrequency TA under direct vision $(54,55,57)$. Hence, PEDTA appears to provide better results than other procedures, as it can significantly reduce intradiscal pressure, remove the inflammed nucleus pulposus under direct vision, provide the TA effect, and reduce the presence of inflammatory factors involved in pain through irrigation (52-57).

Figure 3 demonstrates a case of DLBP due to HIZs, a 48-yearold man who had chronic DLBP for approximately 20 years. MR images revealed HIZs at L4-5, and discography showed concordant pain at the same level ; thus, PEDTA was performed under local anesthesia. Before surgery, the patient had LBP graded 2/ 10 on the visual analog scale (VAS). He also had a history of 3-4 episodes of severe LBP requiring sick leave every year. After the surgery, his LBP almost completely resolved. After undergoing strenuous rehabilitation, including hamstring stretching, trunk 

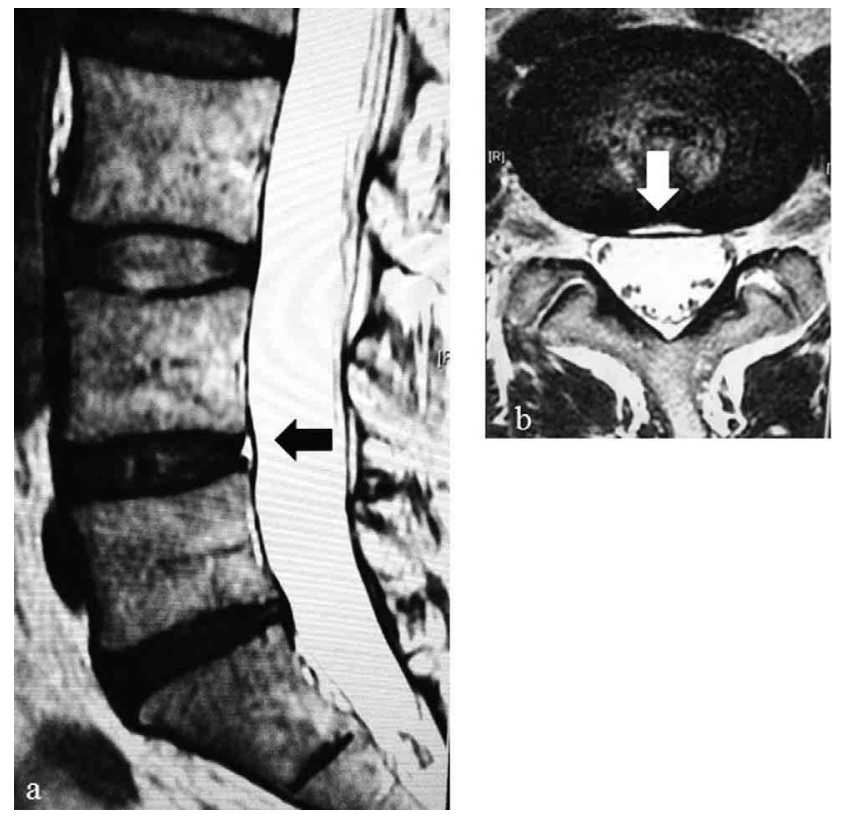

Figure 3. (a) Sagittal and (b) Axial T2-weighted MR images showing HIZs at the LA-5 level (arrows).

muscle core exercises, and thoracic spine stretching, he was able to resume activities of daily living without LBP. Sugiura et al. (57) endoscopically observed the site showing HIZs and found red coloration at the site, suggesting neovascularization into the disc space. Thus, with direct vision of the inflammatory site, TA could be performed as pinpoint surgery that accurately targeted the origin of the pain. Figure 4 shows the MR images of a 36-year-old professional baseball player. He presented with a 1-year history of chronic LBP. The initial MR images (Figure 4a, 4b) revealed herniated nucleus pulposus at the L3-4 and L4-5 levels, with additional HIZs at the L4-5 level. However, provocative discography revealed concordant pain at only the L4-5 level. Hence, discogenic pain due to HIZs at the L4-5 level was diagnosed. Treatment with intradiscal injections was attempted, but little symptomatic relief was achieved. Repeat MR examination (Figure 4c, 4d) after 10 months revealed that the HIZs had become more prominent, with increased intensity at the L4-5 level. PEDTA was therefore performed. Postoperatively, his LBP disappeared, and he returned to playing at the professional level from the next season. At 5 years postoperatively, he has remained very active as a professional baseball player.

PEDTA is a comparatively new technique, and there is not yet much evidence regarding its effects on DLBP. As presented in Figures 3 and 4, PEDTA appears to be highly effective in certain cases. Due to its minimal invasiveness, PEDTA could become the gold standard surgical procedure for DLBP associated with HIZs, following meticulous clinical and basic studies.

\section{CONCLUSION}

$\mathrm{HIZ}$ is not merely a feature of disc degeneration in MR images ; we consider it to be an important sign of painful intervertebral disc disruption, if identified precisely based on factors such as location and intensity. With advances in MR imaging techniques and minimally invasive surgical techniques, further studies are essential for clarifying the controversies regarding the diagnostic significance and management of HIZs.

\section{CONFLICT OF INTERESTS}

The authors declare that there are no conflicts of interests in relation to the article.

\section{ACKNOWLEDGEMENT}

The manuscript submitted does not contain information about medical device(s)/drug(s).

No funds were received in support of this work.
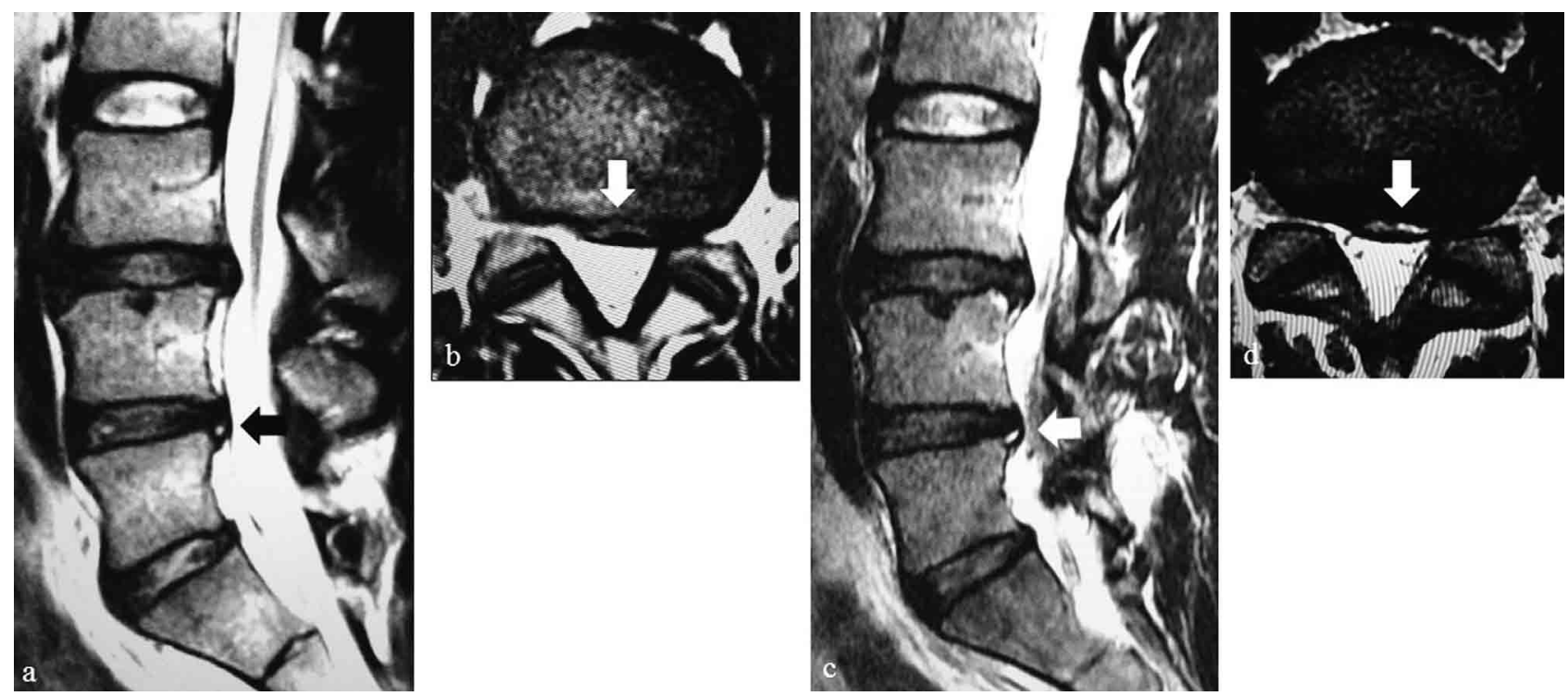

Figure 4. (a and c) Sagittal and ( $b$ and d) axial T2-weighted MR images of the lumbar spine of a professional baseball player. (a and b) MR images at presentation, showing a herniated nucleus pulposus at the L3-4 and L4-5, with additional HIZs at the L4-5 level (arrows). (c and d) Subsequent MR examination performed after 10 months showed increased intensity of HIZs at the LA-5 level (arrows). 


\section{REFERENCES}

1. Airaksinen O, Brox JI, Cedraschi C, Hildebrandt J, KlaberMoffett J, Kovacs F, Mannion AF, Reis S, Staal JB, Ursin H, Zanoli G : European guidelines for the management of chronic nonspecific low back pain. Eur Spine J 15 (Suppl. 2) : S192300, 2006

2. Koes BW, van Tulder MW, Thomas S : Diagnosis and treatment of low back pain. BMJ $332:$ 1430-4, 2006

3. Schwarzer AC, Aprill CN, Derby R, Fortin J, Kine G, Bogduk $\mathrm{N}$ : The prevalence and clinical features of internal disc disruption in patients with chronic low back pain. Spine (Phila Pa 1976) $20: 1878-83,1995$

4. Kallewaard JW, Terheggen MA, Groen GJ, Sluijter ME, Derby R, Kapural L, Mekhail N, van Kleef M : Discogenic low back pain. Pain pract $10: 560-79,2010$

5. Ito M, Iucorvaia KM, Yu SF, Fredrickson BE, Yuan HA, Rosenbaum AE : Predictive signs of discogenic lumbar pain on magnetic resonance imaging with discography correlation. Spine (Phila Pa 1976) $23: 1252-60,1998$

6. Mooney $\mathrm{V}$ : Where is the pain coming from? Spine $8: 754-9$, 1987

7. Laslett M, McDonald B, Tropp H, Aprill CN, Oberg B : Agreement between diagnoses reached by clinical examination and available reference standards : a prospective study of 216 patients with lumbopelvic pain. BMC Musculoskelet Disord $6: 28,2005$

8. Burke JG, Watson RW, McCormack D, Dowling FE, Walsh MG, Fitzpatrick JM : Intervertebral disc which cause low back pain secrete high levels of proinflammatory mediators. J Bone Joint Surg Br 4 : 196-201, 2002

9. Lindbolm K: Diagnostic puncture of intervertebral disks in sciatica. Acta Orthop Scand $17: 231-9,1948$

10. Hirsch $\mathrm{C}:$ An attempt to diagnose level of disc lesion clinically by disc puncture. Acta Orthop Scand 18: 132-40, 1948

11. Provenzano DA : Diagnostic Discography : What is the clinical utility? Curr Pain Headache Rep 16 : 26-34, 2012

12. Wetzel FT : Point of view. The rate of false-positive lumbar discography in select patients without low back symptoms. Spine (Phila Pa 1976) $25: 1381,2000$

13. Aprill C, Bogduk N : High-intensity zone : a diagnostic sign of painful lumbar disc on magnetic resonance imaging. Br J Radiol 65 : 361-9, 1992

14. Saifuddin A, Braithwaite I, White J, Taylor BA, Renton P : The value of lumbar spine magnetic resonance imaging in the demonstration of annular tears. Spine $23: 453-7,1998$

15. Schellhas KP, Pollei SR, Gundry CR, Heithoff KB : Lumbar disc high-intensity zone. Correlation of magnetic resonance imaging and discography. Spine $21: 79-86,1996$

16. Wang ZX, Hu YG : High-intensity zone (HIZ) of lumbar intervertebral disc on $\mathrm{T} 2$-weighted magnetic resonance images : spatial distribution, and correlation of distribution with low back pain (LBP). Eur Spine J 21 : 1311-5, 2012

17. Kaiser JA : Point of view. Lumbar disc high-intensity zone. Correlation of magnetic resonance imaging and discography. Spine $21: 86,1996$

18. Liu C, Cai HX, Zhang JF, Ma JJ, Lu YJ, Fan SW : Quantitative estimation of the high-intensity zone in the lumbar spine : comparison between the symptomatic and asymptomatic population. Spine J 14(3) : 391-396, 2014

19. Bogduk N : Point of view. Predictive signs of discogenic lumbar pain on magnetic resonance imaging with discography correlation. Spine $23: 1259-60,1998$

20. Lam KS, Carlin D, Mulholland RC : Lumbar disc high-intensity zone : the value and significance of provocative discography in the determination of the discogenic pain source. Eur Spine
J 9(1) : 36-41, 2000

21. Rankine JJ, Gill KP, Hutchinson CE, Ross ER, Williamson JB : The clinical significance of the high intensity zone on lumbar spine magnetic resonance imaging. Spine (Phila Pa 1976) $24: 1913-1919,1999$

22. Mitra D, Cassar-Pullicino VN, McCall IW : Longitudinal study of high intensity zones on MR of lumbar intervertebral discs. Clin Radiol 59(11) : 1002-8, 2004

23. Carragee EJ, Paragioudakis SJ, Khurana S : 2000 Volvo Award winner in clinical studies : Lumbar high intensity zone and discography in subjects without low back problems. Spine 25 : 2987-92, 2000

24. Weishaupt D, Zanetti M, Hodler J, Boos N : MR imaging of the lumbar spine : prevalence of intervertebral disk extrusion and sequestration, nerve root compression, end plate abnormalities, and osteoarthritis of the facet joints in asymptomatic volunteers. Radiology 209 : 661-6, 1998

25. Stadnik TW, Lee RR, Coen HL, Neirynck EC, Buisseret TS, Osteaux MJ : Annular tears and disk herniation : prevalence and contrast enhancement on MR images in the absence of low back pain or sciatica. Radiology $206: 49-55,1998$

26. Hebelka H, Hanson T: HIZ's relation to axial load and low back pain : investigation with axial loaded MRI and pressure controlled discography. Eur Spine J 22 : 734-9, 2013

27. Smith B, Hurwitz E, Solsberg D, Rubinstein D, Corenman DS, Dwyer AP, Kleiner J : Interobserver reliability of detecting lumbar intervertebral disc high-intensity zone on magnetic resonance imaging and association of high intensity zone with pain and annular disruption. Spine 23(19) : 207480, 1998

28. Rozen D : Discogenic low back pain. Pain Pract 1 : 278-86, 2010

29. Peng B, Zhang Y, Hou S, Wu W, Fu X : Intradiscal methylene blue injection for the treatment of chronic discogenic low back pain. Eur Spine J $16: 33-8,2007$

30. Peng B, Pang X, Wu Y, Zhao C, Song X : A randomized placebo-controlled trail of intradiscal methylene blue injection for the treatment of chronic discogenic low back pain. Pain $149: 124-9,2010$

31. Boden SD, Davis DO, Dina TS, Patronas NJ, Wiesel SW : Abnormal magnetic resonance scans of the lumbar spine in asymptomatic subjects. A prospective investigation. J Bone Joint Surg Am 72(3) : 403-8, 1990

32. Bogduk N, Tynan W, Wilson AS : The nerve supply to the human lumbar intervertebral disc. J Anat $132: 39-56,1981$

33. Palmgren T, Grondlad M, Virri J, Kaapa E, Karaharju E : An immunohistochemical study of nerve structures in the annulus fibrosus of human normal intervertebral discs. Spine (Phila Pa 1976) 24 : 2075-9, 1999

34. Garcia-Cosamalon J, del Valle ME, Calavia MG, Garcia-Suarez O, Lopez-Muniz A, Otero J, Vega JA : Intervertebral disc, sensory nerves and neurotrophins : who is who in discogenic pain? J Anat $217: 1-15,2010$

35. Sachs BL, Vanharanta H, Spivey MA, Guyer RD, Videman T, Rashbaum RF, Johnson RG, Hochschuler SH, Mooney V: Dallas Discogram description : A new classification of CT/ discography in low back disorders. Spine $12: 287-94,1987$

36. Wang ZX, Hu YG : Clinical investigation of high-intensity zone in anterior annulus fibrosus of lumbar disc : compared with high-intensity zone in posterior annulus fibrosus. Zhonghua Wai Ke Za Zhi 47 : 689-692, 2009

37. Ricketson R, Simmons JW, Hauser BO : The prolapsed intervertebral disc. The high-intensity zone with discography correlation. Spine (Phila Pa 1976) 21(23) : 2758-62, 1996

38. Weidenbaum M, Foster RJ, Best BA, Saed-Nejad F, Nickoloff E, Newhouse J, Ratcliffe A, Mow VC : Correlating magnetic 
resonance imaging with the biochemical content of the normal human intervertebral disc. J Orthop Res 10 : 552-561, 1992

39. Ross JS, Modic MT, Masaryk TJ : Tears of the annulus fibrosus : assessment with Gd-DTPA-enhanced MR imaging. AJR Am J Roentgenol 154 : 159-162, 1990

40. Yu SW, Sether LA, Ho PS, Wagner M, Haughton VM : Tears of the annulus fibrosus : correlation between MR and pathological findings in cadavers. AJNR Am J Neuroradiol 9 : 367 370, 1988

41. Peng B, Hou S, Wu W, Zhang C, Yang Y : The pathogenesis and clinical significance of a high intensity zone (HIZ) of lumbar intervertebral disc on MR imaging in the patient with discogenic low back pain. Eur Spine J 15 : 583-587, 2006

42. Pande KC, Khurjekar K, Kanikdaley V : Correlation of low back pain to a high-intensity zone of the lumbar disc in Indian Patients. J Orthop Surg 17 : 190-193, 2009

43. Wilkens P, Storheim K, Scheel I, Berg L, Espeland A : No effect of 6 -month intake of glucosamine sulfate on modic changes or high intensity zones in the lumbar spine : subgroup analysis of a randomized controlled trial. J Negat Results Biomed $11: 13,2012$

44. Kang CH, Kim YH, Lee SH, Derby R, Kim JH, Chung KB, Sung DJ : Can magnetic resonance imaging accurately predict concordant pain provocation during provocative disc injection? Skeletal Radiol $38: 877-85,2009$

45. O'Neil C, Kurgansky M, Kaiser J, Lau W : Accuracy of MRI for diagnosis of discogenic pain. Pain Physician $11: 311$ 26, 2008

46. Largo R, Alvarez-Soria MA, Diez-Ortego I, Calvo E, SanchezPernaute O, Egido J, Herrero-Beaumont G : Glucosamine inhibits IL-1beta-induced NFkappaB activation in human osteoarthritic chondrocytes. Osteoarthritis Cartilage $11: 290$ 298, 2003

47. Wilkens P, Scheel IB, Grundnes O, Hellum C, Storheim K : Effect of glucosamine on pain-related disability in patients with chronic low back pain and degenerative lumbar osteoarthritis : a randomized controlled trial. JAMA $304: 45-52,2010$

48. Miller MR, Mathews RS, Reeves KD : Treatment of painful advanced internal lumbar disc derangement in intradiscal injection of hypertonic dextrose. Pain Physician 9 : 115-121, 2006

49. Derby R, Eek B, Lee SH, Seo KS, Kim BJ : Comparison of intradiscal restorative injections and intradiscal electrothermal treatment (IDET) in the treatment of low back pain. Pain Physician 7 : 63-66, 2004

50. Kvarstein G, Mawe L, Indahl A, Hol PK, Tennoe B, Digernes R, Stubhaug A, Tonnessen TI, Beivik H : A randomized doubleblind controlled trial of intra-annular radiofrequency thermal disc therapy-a 12-month follow-up. Pain 145 : 279-286, 2009

51. Narvani AA, Tsiridis E, Wilson LF : High-intensity zone, intradiscal electrothermal therapy, and magnetic resonance imaging. J Spinal Disord Tech $16: 130-136,2003$

52. Yeung AT, Tsou PM : Posterolateral endoscopic excision for lumbar disc herniation. Spine 27 : 722-731, 2002

53. Sairyo K, Egawa H, Matsuura T, Takahashi M, Higashino K, Sakai T, Suzue N, Hamada D, Goto T, Takata Y, Nishisho T, Goda Y, Sato R, Tsutsui T, Tonogai I, Kondo K, Tezuka F, Mineta K, Sugiura K, Takeuchi M, Dezawa A: State of the art : Transforaminal approach for percutaneous endoscopic lumbar discectomy under local anesthesia. J Med Invest 61 : 217-225, 2014

54. Tsou PM, Alan Yeung C, Yeung AT : Posterolateral transforaminal selective endoscopic discectomy and thermal annuloplasty for chronic lumbar discogenic pain : a minimal access visualized intradiscal surgical procedure. Spine J 4 : 564-573, 2004

55. Sairyo K, Kitagawa Y, Dezawa A : Percutaneous endoscopic discectomy and thermal annuloplasty for professional athletes. Asian J Endosc Surg 6 : 292-297, 2013

56. Cheng J, Zheng W, Wang H, Li C, Wang J, Zhang Z, Zhou Y : Posterolateral transforaminal selective endoscopic discectomy with thermal annuloplasty for discogenic low back pain. Spine (Phila Pa 1976) 39 : B60-B65, 2014

57. Sugiura K, Tonogai I, Matsuura T, Higashino K, Sakai T, Suzue N, Hamada D, Goto T, Takata Y, Nishisho T, Goda Y, Sato R, Kondo K, Tezuka F, Mineta K, Takeuchi M, Takahashi M, Egawa H, Sairyo K : Discoscopic findings of high intensity zones on magnetic resonance imaging of lumbar intervertebral discs. Case Rep Orthop 2014 